\title{
On the role of oxygen in fabricating microfluidic channels with ultraviolet curable materials ${ }^{\dagger}$
}

\author{
Hoon Eui Jeong ${ }^{a}$ and Kahp Y. Suh*a,b \\ Received 18th June 2008, Accepted 1st September 2008 \\ First published as an Advance Article on the web 11th September 2008 \\ DOI: $10.1039 / b 810348 h$
}

We present the effects of oxygen on the irreversible bonding of a microchannel using an ultraviolet (UV) curable material of polyurethane acrylate (PUA). Microchannels were fabricated by bonding a top layer with impressions of a microfluidic channel and a bottom layer consisting of a PUA coating on a glass or a polyethylene terephthalate (PET) film substrate. The resulting channel is a homogeneous conduit of the PUA material. To find optimal bonding conditions, the bottom layer was cured under different oxygen concentration and UV exposure time at a constant UV intensity $\left(10 \mathrm{~mW} \mathrm{~cm}^{-2}\right)$. Our experimental and theoretical studies revealed that the channel bonding is severely affected by the concentration of oxygen either in the form of trapped air or permeated air out of the channel. In addition, an optimal UV exposure time is needed to prevent clogging or non-bonding of the channel.

\section{Introduction}

Poly(dimethylsiloxane) (PDMS) is one of the most widely used polymeric materials for fabricating microfluidic devices because it allows for easy manufacturing by rapid prototyping and favorable mechanical and optical properties such as softness, transparency and low autofluorescence. ${ }^{1,2}$ In spite of these advantages, it takes more than $1 \mathrm{~h}$ to cure PDMS at an elevated temperature $\left(\sim 70{ }^{\circ} \mathrm{C}\right)$. Also, PDMS channels are typically hydrophobic (contact angle of water $\sim 105^{\circ}$ ), which causes nonspecific adsorption of biological species, ${ }^{3,4}$ limited capillary flow inside channels, ${ }^{5,6}$ and poor chemical compatibility with many organic solvents. ${ }^{7}$ Moreover, the fabrication of a nanoscale channel is not easily achieved with PDMS due to low elastic modulus $(\sim 1.8 \mathrm{MPa})$ of the material. ${ }^{8}$ Rigid materials such as silicon and glass can overcome some of the shortcomings of PDMS, but their widespread use is limited by complicated and expensive fabrication process, intrinsic stiffness, low biocompatibility, and biofouling., ${ }^{1,2,-11}$ In addition to the above mentioned materials, various polymeric materials have been introduced, including poly(carbonate) (PC), ${ }^{12-14}$ poly(methyl methacrylate) (PMMA), ${ }^{15,16}$ polyimide (PI), ${ }^{17,18}$ parylene-C, ${ }^{19}$

${ }^{a}$ School of Mechanical and Aerospace Engineering, Seoul National University, Seoul, 151-742, Korea

${ }^{b}$ The Institute of Bioengineering, Seoul National University, Seoul, 151-742, Korea.E-mail: sky4u@snu.ac.kr

$\dagger$ In honour of Andreas Manz on his retirement as Chair of the Lab on a Chip Editorial Board.
SU- $8,{ }^{20}$ poly(ethylene glycol) (PEG),${ }^{5,21,22}$ NOA (Norland optical adhesive $)^{23,24}$ and poly(urethane acrylate) (PUA) ${ }^{25}$

Of these, UV curable materials have several advantages, such as rapid prototyping with short curing time, low energy consumption, room temperature operation, excellent resistance toward organic solvents/chemicals/heats and tunable properties of the polymers. ${ }^{26}$ With these advantages, microfluidic devices have been successfully fabricated by using UV curable resins such as PEG diacrylate (PEG-DA), ${ }^{21}$ PEG dimethacrylate (PEGDMA), ${ }^{21} \mathrm{NOA}^{23,24} \mathrm{PUA}^{25}$ and poly(vinyl silazane). ${ }^{27}$ Generally, microchannels are fabricated by bonding partially cured top and bottom layers aided by an elevated temperature and/or an applied pressure. In the photocrosslinking step, it is known that oxygen inhibits free radical polymerization by scavenging initiating radicals when the surface of the resin is exposed to air. As a result, the surface remains undercured and tacky, ${ }^{26}$ which enables subsequent bonding of the two layers. Although several methods have been developed to deal with oxygeninduced partial curing, mainly in the areas of thin film coating, ${ }^{28}$ researchers are now utilizing the oxygen inhibitory effect for fabricating micro- and nanoscale channels, ${ }^{5,21,23}$ micro/nanoscale hierarchical structures ${ }^{29}$ and generating microparticles inside microfluidic devices. ${ }^{30,31}$ In response to increasing demands in finding optimal curing conditions of the UV curable materials, it would be beneficial to study the role of oxygen inhibition in the irreversible sealing of microchannels.

Here, we analyze the oxygen inhibitory effects on the photocrosslinking and subsequent channel bonding by changing oxygen concentration and curing time with an UV curable polyurethane acrylate (PUA) resin. The concentration of oxygen in contact with the PUA resin was controlled by using different blanket layers (no blanket, PDMS, and PUA) with different permeability to air. Our experimental results demonstrate that the oxygen can assist complete bonding of channels either in the form of trapped air or permeated air out of the channel. Also, an optimal curing time is needed to induce a robust bonding. To explain our experimental observations, a simple theoretical model was developed based on one-dimensional kinetic equation by incorporating competition between oxygen consumption and permeation.

\section{Materials and methods}

\section{Fabrication of PUA microchannels}

A small amount $(\sim 100 \mu \mathrm{L})$ of UV curable PUA (Minuta Co., Korea) was drop-dispensed on a silicon master and a supporting polyethylene terephthalate (PET) film was placed on top of the 
PUA drop followed by UV exposure $(\sim 250-400 \mathrm{~nm})$ for $20 \mathrm{~s}$ through the transparent PET film at an intensity of $10 \mathrm{~mW} \mathrm{~cm}^{-2}$. The PET film used in this process was surface modified with urethane groups to increase adhesion to the acrylate-containing monomer (Sun Chemical Co., Korea). The silicon master was prepared by photolithography, having protruding (positive) features with impressions of a microfluidic channel $(145 \mu \mathrm{m}$ width and $45 \mu \mathrm{m}$ height). After UV curing, the crosslinked PUA channel mold (top layer of channel) was peeled off from the master and both ends of the channel were cut, leaving behind an open-ended channel. All the top layers were exposed to UV for the same duration of time ( $20 \mathrm{~s})$. The PUA channel mold was then brought in conformal contact with a bottom layer of glass slide or PET film with a PUA coating (thickness $\sim 10 \mu \mathrm{m})$. The two layers were bonded with an additional UV exposure for several minutes under a slight pressure $\left(\sim 10^{3} \mathrm{~Pa}\right)$. The PUA resin on the bottom layer was cured for $5 \mathrm{~s}$ to $30 \mathrm{~min}$ depending on blanket materials (no blanket, PDMS blanket and PET blanket). Here, the blankets were used for changing oxygen concentration in contact with the PUA surface. By utilizing the different permeabilities of the blanket materials to air, the degree of crosslinking was modulated by controlling UV exposure time.

\section{Fabrication of microchannels with rhodamine-containing PUA bottom layer}

A top layer of the channel was fabricated using the same procedure described above. Here, a Y-shaped channel with one inlet and two outlets was used for introducing DI water. After UV curing, the crosslinked PUA channel mold (top layer of channel) was peeled off from the master and both ends of the channel were cut, leaving behind an open-ended channel for capillarity.

For fabricating a bottom layer of the channel, a small amount $(\sim 100 \mu \mathrm{L})$ of the PUA resin containing $0.5 \mathrm{wt} \%$ rhodamine was drop-dispensed on a glass slide and a flat PDMS block was placed on top. The rhodamine was mixed with the PUA to visualize elution of undercured PUA resin. Then, the half channel covered with the PDMS block was exposed to UV for $25 \mathrm{~s}$ whereas the other half channel was exposed for $50 \mathrm{~s}$. The top PUA channel mold was then aligned with the bottom layer and bonded with an additional UV exposure for several minutes. After fabricating the microchannel, an elution behavior of the PUA resin was examined over time by flowing DI water through the inlet of the channel by capillarity using an inverted fluorescence microscope (OLYMPUS IX71, Japan).

\section{Scanning electron microscopy (SEM)}

Scanning electron microscopy (SEM) images of the PUA microchannels were obtained using a XL30FEG microscope (Philips Electron Co., Netherlands) operating at an accelerating voltage of $5 \mathrm{kV}$. Samples were coated with a $10 \mathrm{~nm}$ Pt layer prior to measurements to prevent charging effects.

\section{Nanoindentation}

The elastic modulus of the cured PUA layer was measured using a nanoindenter (Nano Indenter XP, MTS). The target depth was $700 \mathrm{~nm}$, and the typical mean load on the bone was $0.2-1.0 \mathrm{mN}$.
The presented values were measured over at least 10 points per sample.

\section{Results and discussion}

\section{Effects of oxygen inhibition and UV exposure time on the fabrication of microchannels}

The irreversible bonding procedure with the UV curable PUA material is shown in Scheme 1. In the bonding step, the key mechanism is a partial curing of the bottom layer, which is mainly affected by the concentration of oxygen that is in contact with the resin surface. ${ }^{26,29}$ To determine the effects of oxygen on UV radiation curing during the microchannel fabrication, the bottom layers of the channel were cured in ambient air without a blanket or by using a blanket with different permeability to air (PDMS and PET film) (Scheme 1). In addition, various UV exposure times were tested to find an optimal curing time. After curing, the top layer of the channel was brought in conformal contact with the bottom layer followed by subsequent UV exposure for complete sealing.

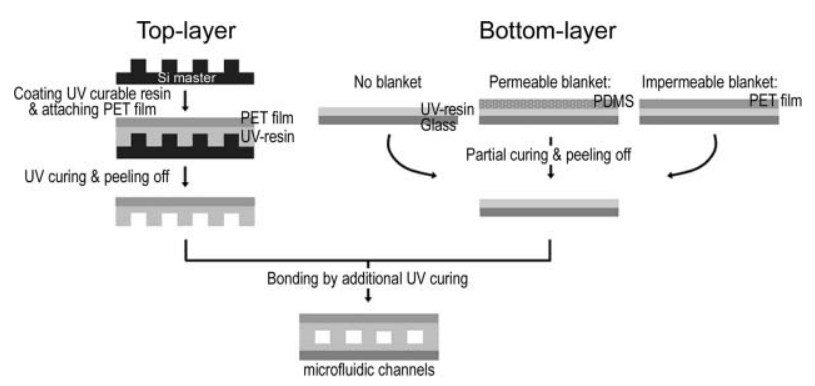

Scheme 1 Schematic diagram of the procedure for channel fabrication. A top layer of channel was prepared by replica moulding against a silicon master with PUA resin. A bottom layer of channel was cured under three different conditions: the surface resin was directly exposed to atmospheric air or covered with two different film materials having different permeability to air (PDMS and PET film). After fabricating top- and bottom layers, the two layers were brought in contact with a slight pressure, followed by an additional UV exposure for irreversible sealing.

Fig. 1 shows cross-sectional scanning electron microscopy (SEM) images and top-view optical microscopy images of various microchannels fabricated under different curing conditions. For this experiment, microchannels of $145 \mu \mathrm{m}$ in width and $45 \mu \mathrm{m}$ in height were used. It was found that the PUA resin was barely cured without a blanket layer (Fig. 1a), unless the curing time was longer than $30 \mathrm{~min}$. As shown in the figure, when the curing time was $5 \mathrm{~min}$, the uncured PUA resin filled into the cavity and clogged the channel. For an increased curing time to $15 \mathrm{~min}$, the PUA was still undercured and seeped into the channel. Although a relatively robust bonding was obtained when the curing time was enhanced to $30 \mathrm{~min}$, there still was an undercured region, resulting in partial filling into the channel as shown in Fig. 1a. This indicates that the minimum curing time should be $>30 \mathrm{~min}$ in the absence of a blanket layer. This is presumably due to the fact that the atmospheric oxygen steadily diffuses into the PUA surface and replaces the oxygen consumed by free radicals. Although a long exposure time might lead to better results, the advantage of UV-curable materials, i.e., rapid 


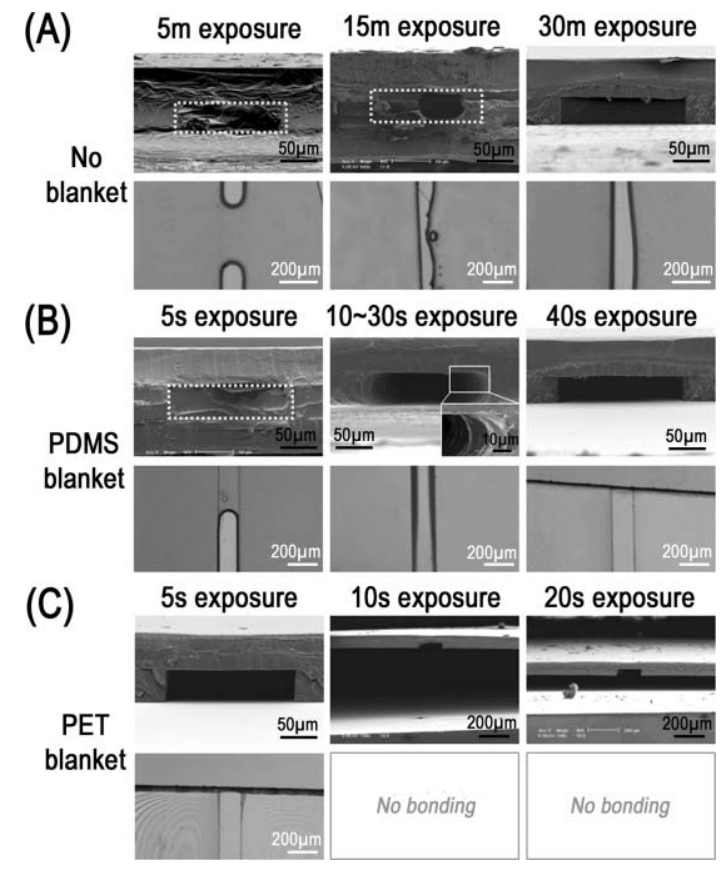

Fig. 1 (A-C) Cross-sectional SEM images and corresponding topview optical microscopy images of the microchannels fabricated under different conditions. The bottom layers were cured with (A) no blanket, (B) PDMS blanket, and (C) PET blanket under different UV exposure time.

curing within a few seconds, would be significantly diminished. Therefore, an oxygen barrier appears essential to induce a robust channel bonding by lowering the concentration of oxygen to a required level. It is noted in this regard that the concentration of dissolved oxygen has to decrease by at least two orders of magnitude for initiating polymerization of acrylate double bonds. ${ }^{26}$

Fig. 1b shows results when a PDMS blanket was used as a diffusion barrier. Since the PDMS is highly permeable (permeability of PDMS $\sim 3.6 \times 10^{-2} \mu \mathrm{m}^{2} \mathrm{~Pa}^{-1} \mathrm{~s}^{-1}$ ) and protects the surface from direct exposure to air ${ }^{32}$ the polymerization level of the PUA resin can be controlled by changing UV exposure time. Our experimental results demonstrated that at least $10 \mathrm{~s}$ exposure time is needed with a PDMS blanket to avoid clogging of the channel. Under $10 \mathrm{~s} \mathrm{UV}$ irradiation, the PUA was not cured and filled into the channel. With increasing UV exposure time (10-30 s), the polymerization of PUA resin further proceeded and the channel bonding was completed without failure. Based on our experimental results, the appropriate irradiation time was between 40 to $50 \mathrm{~s}$, as judged by the cross-sectional SEM and microscopy images in Fig. 1b. With optimized UV exposure time, only the surface of the PUA resin in contact with air remains tacky, whereas the resin beneath the surface cures completely, resulting in robust channels without a notable distortion or rounding of the channels.

Channel distortions generally pose a number of problems in manipulating flow inside a channel, handling biological species (proteins or cells) or preventing a partial leak at the interface. These problems would become more prominent as the channel dimension goes smaller. Therefore, a slight distortion or rounding at the corner in Fig. 1 might be detrimental to the fabrication of smaller channels (e.g., nanochannels) and their performance as an analytical device. In terms of optimal curing times, however, there would be no notable change for smaller channels since the bottom layer of the channel was partially cured and the top layer of the channel was just added on top (see Scheme 1). A further study would be required to address this issue in detail.

We also tested a PET film as a diffusion barrier against oxygen. The permeability of the PET film is about 6 orders of magnitude lower than that of the PDMS (permeability of the PET film of $500 \mu \mathrm{m}$ thickness $\left.\sim 2.7 \times 10^{-8} \mu \mathrm{m}^{2} \mathrm{~Pa}^{-1} \mathrm{~s}^{-1}\right){ }^{33}$ It was found that the UV exposure time should be shorter than $5 \mathrm{~s}$ for a robust bonding of microchannels. When the curing time was $>5 \mathrm{~s}$, the top and $\mathrm{e}$ layers were not bonded, as shown in Fig. 1c. It is worthwhile noting that even for a very short exposure time $(<5 \mathrm{~s})$, the bonding strength of the channel was quite weak, such that the top and bottom layers of the channel were easily separated when a PET film was used as the blanket. It is because the PUA resin rapidly cures within $5 \mathrm{~s}$, as there is no permeated air through the impermeable PET blanket. Moreover, there is little trapped air between the PET blanket and the surface of resin without a gap. As a consequence, the use of a PDMS blanket would be useful to achieve robust bonding of microchannels by tailoring the degree of partial curing with time.

The tacky nature can be characterized by measuring mechanical rigidity of the partially cured surface by nanoindentation. It was observed that the elastic modulus of the cured top surface increased with increasing curing time with or without a blanket, but with different speeds (Fig. 2). In the absence of a blanket, the modulus gradually increased and reached $\sim 0.9$ $\mathrm{GPa}$ after $2400 \mathrm{~s}$, which is still far below the bulk modulus $(\sim 2.5 \mathrm{GPa})$. This indicates that the top surface is not completely cured and partially fluidic. In the case of a PDMS blanket, the modulus increased relatively faster than that without a blanket and reached $\sim .7 \mathrm{GPa}$ after $180 \mathrm{~s}$, suggesting that the photocrosslinking is inhibited by oxygen. In the case of a PET blanket, the modulus rapidly increased to the bulk modulus after $60 \mathrm{~s}$. Based on our optimal curing times for complete sealing with or without a blanket, the modulus needs to be controlled within a range between 0.7 and $1.2 \mathrm{GPa}$ for the materials and UV intensity used in our experiment. Similarly, the elastic modulus could be used as an indicator to find optimal bonding conditions for other UV curable materials.

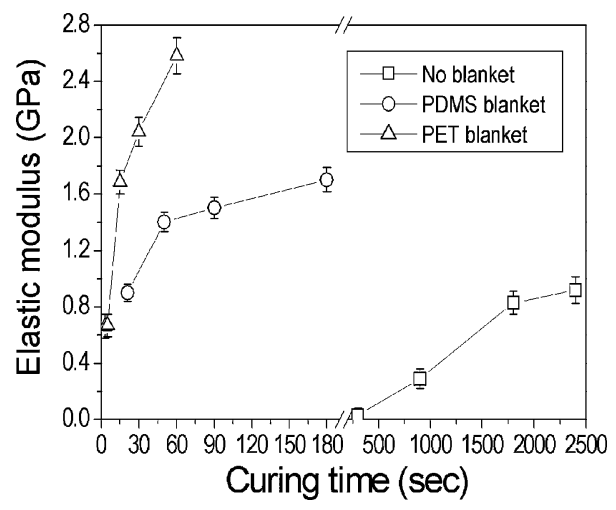

Fig. 2 Measurements of the elastic modulus of the cured PUA resin with UV curing time by nanoindentation. 


\section{Analysis of change of oxygen concentration on the resin surface}

Our experimental results indicate that the minimum curing time for robust bonding depends on the permeability of blanket materials. For example, the minimum curing time for the PET blanket ( $5 \mathrm{~s}$ ) was about 8 and 360 times lower than that for the PDMS blanket (40 s) and no blanket (30 min), respectively.

To gain an understanding on the curing kinetics during the fabrication of the bottom layer of channel, we consider a onedimensional kinetic equation for oxygen for the PET and PDMS blankets, respectively.

$$
\begin{aligned}
-\frac{\mathrm{d} C_{\mathrm{O}_{2}}}{\mathrm{~d} t} & =k C_{\mathrm{O}_{2}} \quad \text { (PET blanket) } \\
-\frac{\mathrm{d} C_{\mathrm{O}_{2}}}{\mathrm{~d} t} & =k C_{\mathrm{O}_{2}}-P \quad \text { (PDMS blanket) }
\end{aligned}
$$

where $C_{\mathrm{O}_{2}}$ is the oxygen concentration remaining between the blanket and the surface of the PUA resin and $P$ is the oxygen concentration permeated through the porous PDMS blanket (see Fig. 3a). Here, the amount of permeated oxygen was assumed constant for a given permeability and a pressure drop between inside and outside of the blanket. Integrating eqn (1) yields

$$
\begin{gathered}
\frac{C_{\mathrm{O}_{2}}}{C_{\mathrm{i}, \mathrm{O}_{2}}}=\exp (-k t)(\text { PET blanket }) \\
\frac{C_{\mathrm{O}_{2}}}{C_{\mathrm{i}, \mathrm{O}_{2}}}=\left(1-\frac{P}{k C_{\mathrm{i}, \mathrm{O}_{2}}}\right) \exp (-k t)+\frac{P}{k C_{\mathrm{i}, \mathrm{O}_{2}}} \text { (PDMS blanket) }
\end{gathered}
$$

where $C_{\mathrm{i}, \mathrm{O}_{2}}$ represents the initial amount of trapped oxygen and $P / k C_{\mathrm{i}, \mathrm{O}_{2}}$ is the dimensionless parameter that can describe the ratio of permeation rate to oxygen consumption rate. Thus, the effects of permeability can be examined by varying the values of $P / k C_{\mathrm{i}, \mathrm{O}_{2}}$. The change of oxygen concentration in contact with the surface of resin is plotted in Fig. $3 \mathrm{~b}$ as a function of time for different values of $P / k C_{\mathrm{i}, \mathrm{O}_{2}}$. In this plot, the value of

(A)

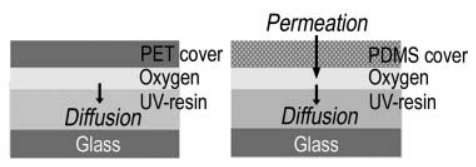

(B)

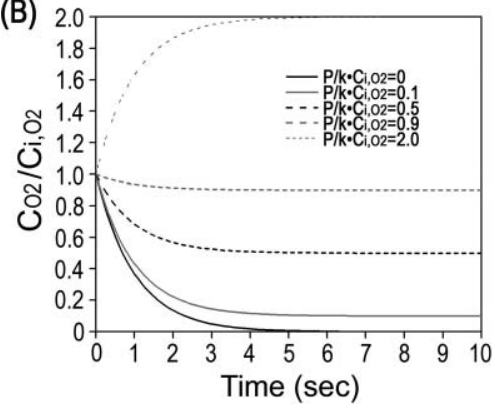

Fig. 3 (A) Schematics for the cross-sectional view of the bottom layer with impermeable PET film (left) and porous PDMS blankets (right). (B) Change of oxygen concentration as a function of time for PET and PDMS blankets with different permeability. The $k$ value was set to 1 in the plots. $k$ was assumed to be 1 , provided that the curing is complete for $0.01<C_{\mathrm{O}_{2}} / C_{\mathrm{i}, \mathrm{O}_{2}}<0.001$. As shown in the figure, when the permeation rate is nearly zero (e.g., a PET blanket), the amount of oxygen beneath the impermeable PET blanket is consumed rapidly within a few seconds. In this case, eqn (3) is reduced to eqn (2) (no permeation). With increasing the ratio of permeation rate to oxygen consumption rate $\left(0<P / k C_{\mathrm{i}, \mathrm{O}_{2}}<1\right)$, the surface of resin is constantly exposed to oxygen for a long period of time (e.g., a PDMS blanket or no blanket with a high UV intensity). If the permeation rate becomes similar to or higher than the oxygen consumption rate $\left(P / k C_{\mathrm{i}, \mathrm{O}_{2}} \geq 1\right)$, the oxygen concentration is maintained at the initial level or even increases with time (e.g., no blanket with a low UV intensity). It was frequently observed that the UV curing was never completed without a blanket at a lower UV intensity than $\sim 0.1 \mathrm{~mW} \mathrm{~cm}^{-2}$ (data not shown).

\section{Elution of undercured UV-resin inside microchannels}

The UV radiation curing is a simple way to transform a liquid resin into a solid polymer within a few seconds without any complicated process. However, the uncured resin molecules could elute from the surface in a solution, recognizing the fact that the UV radiation curing is generally carried out in the presence of air. In fact, microchannels that were fabricated in previous works were prepared while exposed to air. ${ }^{5,21,23,34,35}$ Since UV curable resins are optically transparent, it is not easy to detect the elution in a conventional flow operating condition.

To investigate the elution behavior of UV resin molecules cured for different exposure times (for a given UV intensity and a oxygen permeability) inside microfluidic devices, we fabricated Y-shaped channels having one inlet and two outlets (see Fig. 4a)
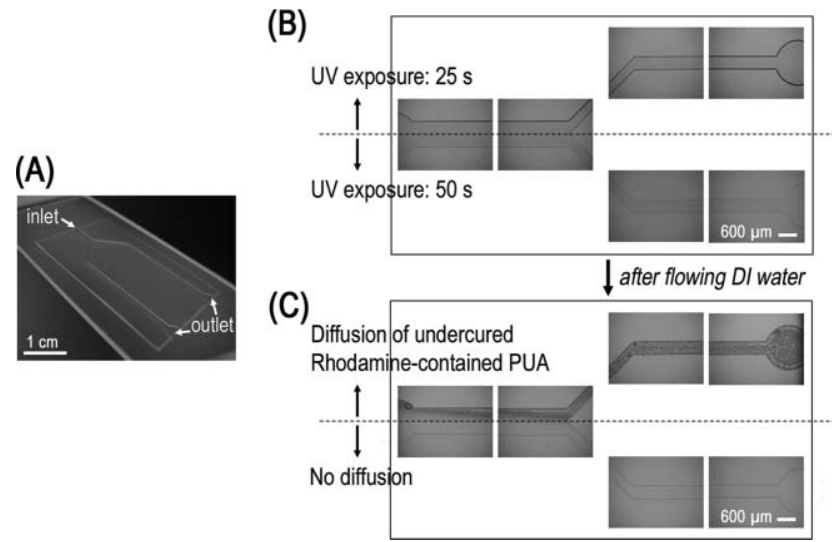

Fig. 4 (A) Y-shaped microchannels having one inlet and two outlets fabricated by partial curing of a bottom layer and subsequent bonding with a top layer. To visualize the elution of undercured PUA resin, rhodamine was mixed with the bottom layer prior to bonding. In the fabrication of the bottom layer, the half channel was exposed to UV for $25 \mathrm{~s}$ and the other half channel was exposed to UV for $50 \mathrm{~s}$ (both sides were covered with a bare PDMS block). (B) Magnified inverted microscopy images at different positions of the channel. Channels were bonded without any notable leaks or distortions. (C) Magnified inverted microscopy images of the microchannel at different positions after flowing DI water through the channel. The half side exposed to UV for $25 \mathrm{~s}$ turns to a deep red due to elution of the undercured rhodaminecontaining PUA resin. The red background colour can be seen in the online version. 
by partial curing of the bottom layer and subsequent bonding process. To visualize the diffusion of the PUA molecules, rhodamine was mixed with the PUA resin for the fabrication of bottom layer of the channel. The top layer of the channel was fabricated with a pure PUA resin by using replica moulding against a silicon master.

Fig. 4b shows the fabricated microfluidic channel along with magnified inverted microscopy images at different positions of the channel. As shown in the figure, it appears that channels were completely bonded without any notable leaks or distortions. After flowing DI water through the inlet of the channel, however, the half channel exposed to UV for a shorter duration of time (25 s) changed to a deep red as a result of diffusion of the rhodamine-containing PUA resin, suggesting that there are undercured, eluting PUA molecules on the surface (see Fig. 4c). On the other hand, no notable changes were observed on the other half channel that had been exposed to UV for a longer duration of time $(50 \mathrm{~s})$. These results reveal that although channels were fabricated with no apparent sealing problems, elution can occur from the undercured surface, unless curing conditions were not carefully optimized. When small sample quantities such as rare proteins are involved, these eluting molecules could result in a critical error in the final analysis. In addition, the diffusion of undercured resin could contaminate the channel or compromise cell viability. In this regard, the optimization of curing process enables robust channel fabrication as well as precise analysis in diagnostic and cell-based devices.

\section{Conclusions}

We have studied the role of oxygen in the fabrication of microfluidic channels aided by partial curing of the UV curable PUA material. The effects of oxygen on the channel fabrication have been analyzed by changing oxygen concentration and UV curing time. Several notable findings were derived from the experiment. First, the bonding event was heavily affected by the concentration of oxygen. When the surface of resin was directly exposed to air without any blanket (high concentration), the amount of air diffusing into the resin surface was so large that the photocrosslinking was much delayed $(>30 \mathrm{~min})$ or never completed. When a permeable PDMS blanket was used (intermediate concentration), it assisted irreversible bonding by rendering the resin surface tacky and partially fluidic within a reasonable duration of time (40-50 s). When an impermeable PET film was used as a blanket (low concentration), the PUA resin rapidly cured within $5 \mathrm{~s}$, resulting in less controllability or a failure of robust channel bonding.

In addition to the oxygen concentration, various UV exposure times were tested to find an optimal curing time. When the curing time was too short, the channel was collapsed by capillary filling of the resin. Furthermore, the undercured resin molecules eluted from the surface in a solution. When the curing time was too long, on the other hand, the bonding failed due to non-fluidic nature of the surface. These findings would offer a guide to optimize bonding conditions for fabricating robust micro- and nanofluidic channels with UV curable materials.

\section{Acknowledgements}

This work was supported by the Korea Science \& Engineering Foundation through the Nano R \& D Program (Grant 200702605) and by the Center for Nanoscale Mechatronics \& Manufacturing (Grant 08K1401-00210) under the auspices of the Ministry of Education, Science and Technology, KOREA. This work was also supported in part by the Korea Research Foundation Grant funded by the Korean Government (MOEHRD) (Grant KRF-J03000).

\section{References}

1 D. C. Duffy, J. C. McDonald, O. J. A. Schueller and G. M. Whitesides, Anal. Chem., 1998, 70, $4974-4984$.

2 T. J. Johnson, D. Ross, M. Gaitan and L. E. Locascio, Anal. Chem., 2001, 73, 3656-3661.

3 V. Linder, E. Verpoorte, W. Thormann, N. F. de Rooij and M. Sigrist, Anal. Chem., 2001, 73, 4181-4189.

4 G. Ocvirk, M. Munroe, T. Tang, R. Oleschuk, K. Westra and D. J. Harrison, Electrophoresis, 2000, 21, 107-115.

5 H. E. Jeong, P. Kim, M. K. Kwak, C. H. Seo and K. Y. Suh, Small, 2007, 3, 778-782.

6 E. Kim, Y. N. Xia and G. M. Whitesides, J. Am. Chem. Soc., 1996, 118, 5722-5731.

7 J. N. Lee, C. Park and G. M. Whitesides, Anal. Chem., 2003, 75, 6544-6554.

8 C. Y. Hui, A. Jagota, Y. Y. Lin and E. J. Kramer, Langmuir, 2002, 18, 1394-1407.

9 D. J. Harrison, A. Manz and Z. H. Fan, Anal. Chem., 1992, 64, 1926-1932.

10 J. Lahann, M. Balcells, H. Lu, T. Rodon, K. F. Jensen and R. Langer, Anal. Chem., 2003, 75, 2117-2122.

11 K. C. Popat, R. W. Johnson and T. A. Desai, J. Vac. Sci. Technol. B, 2003, 21, 645-654.

12 B. R. Flachsbart, K. Wong, J. M. Iannacone, E. N. Abante, R. L. Vlach, P. A. Rauchfuss, P. W. Bohn, J. V. Sweedler and M. A. Shannon, Lab Chip, 2006, 6, 667-674.

13 Y. Kong, H. W. Chen, Y. R. Wang and S. A. Soper, Electrophoresis, 2006, 27, 2940-2950.

14 J. F. Chen, M. Wabuyele, H. W. Chen, D. Patterson, M. Hupert, H. Shadpour, D. Nikitopoulos and S. A. Soper, Anal. Chem., 2005, 77, 658-666.

15 L. Brown, T. Koerner, J. H. Horton and R. D. Oleschuk, Lab Chip, 2006, 6, 66-73.

16 Y. L. Bai, C. G. Koh, M. Boreman, Y. J. Juang, I. C. Tang, L. J. Lee and S. T. Yang, Langmuir, 2006, 22, 94589467.

17 R. Barrett, M. Faucon, J. Lopez, G. Cristobal, F. Destremaut, A. Dodge, P. Guillot, P. Laval, C. Masselon and J. B. Salmon, Lab Chip, 2006, 6, 494-499.

18 E. D. Moss, A. Han and A. B. Frazier, Sens. Actuators, B, 2007, 121, 689-697.

19 T. Y. Chang, V. G. Yadav, S. De Leo, A. Mohedas, B. Rajalingam, C. L. Chen, S. Selvarasah, M. R. Dokmeci and A. Khademhosseini, Langmuir, 2007, 23, 11718-11725.

20 S. Tuomikoski and S. Franssila, Sens. Actuators, A, 2005, 120, 408415.

21 P. Kim, H. E. Jeong, A. Khademhosseini and K. Y. Suh, Lab Chip, 2006, 6, 1432-1437.

22 P. Kim, M. C. Park, K. W. Kwon, S. H. Lee, S. M. Kim and K. Y. Suh, Biochip J., 2008, 2, 1-11.

23 D. Bartolo, G. Degre, P. Nghe and V. Studer, Lab Chip, 2008, 8, 274-279.

24 L.-H. Hung, R. Lin and A. P. Lee, Lab Chip, 2008, 8, 983987.

25 M. C. Park, J. Y. Hur, K. W. Kwon, S. H. Park and K. Y. Suh, Lab Chip, 2006, 6, 988-994.

26 C. Decker, Macromol. Rapid Commun., 2002, 23, 10671093.

27 A. Asthana, Y. Asthana, I. K. Sung and D. P. Kim, Lab Chip, 2006, 6, $1200-1204$. 
28 K. Studer, C. Decker, E. Beck and R. Schwalm, Prog. Org. Coatings, 2003, 48, 92-100.

29 H. E. Jeong, R. Kwak, J. K. Kim and K. Y. Suh, Small, 2008, DOI: $10.1002 / \mathrm{smll} .200800151$.

30 D. Dendukuri, S. S. Gu, D. C. Pregibon, T. A. Hatton and P. S. Doyle, Lab Chip, 2007, 7, 818-828.

31 D. Dendukuri, D. C. Pregibon, J. Collins, T. A. Hatton and P. S. Doyle, Nat. Mater., 2006, 5, 365-369.
32 T. C. Merkel, V. I. Bondar, K. Nagai, B. D. Freeman and I. Pinnau, J. Polym. Sci., Part B: Polym Phys., 2000, 38, 415-434.

33 K. Y. Suh, H. E. Jeong, D. H. Kim, R. A. Singh and E. S. Yoon, J. Appl. Phys., 2006, 100, 034303.

34 Q. Chen, G. Li, Q. H. Jin, J. L. Zhao, Q. S. Ren and Y. S. Xu, J. Microelectromech. Syst., 2007, 16, 1193-1200.

35 S. Y. Lai, X. Cao and L. J. Lee, Anal. Chem., 2004, 76, 11751183. 